\title{
Efficient Therapy Planning via Model Reduction for Laser-Induced Interstitial Thermotherapy
}

\author{
Kevin Tolle, Nicole Marheineke
}

\section{Motivation}

Laser-induced interstitial thermotherapy (LITT) is a minimally invasive, local form of treatment for tumors, which in many cases is more preferable than alternatives such as surgical removal or chemotherapy. Low-powered laser light is applied to the targeted tissue causing coagulation and eventually destroying the tissue.

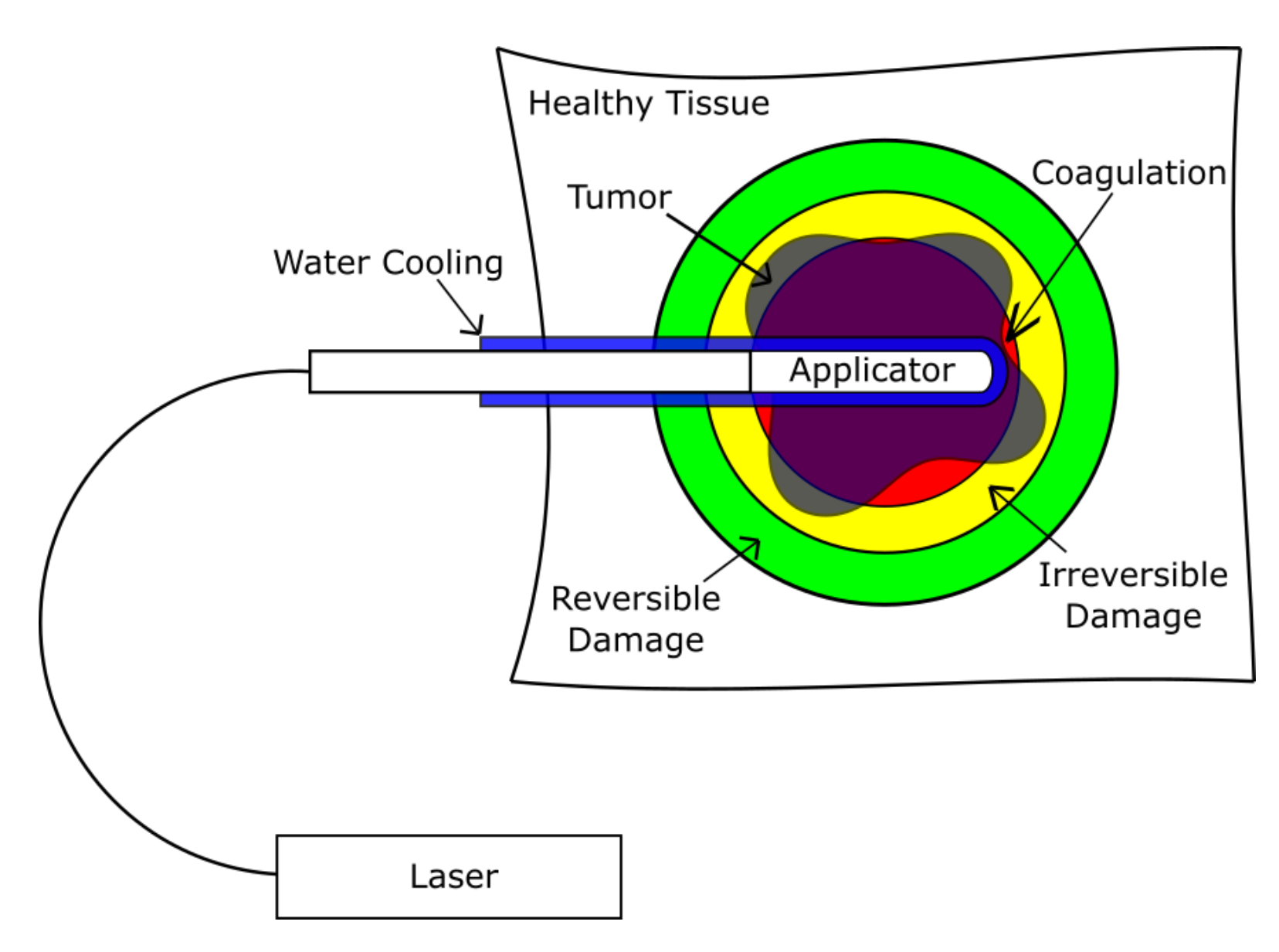

\section{Goal}

Control the laser power, so that the complete destruction of the tumor is ensured, while leaving as much healthy tissue unharmed as possible.

\section{Mathematical Model}

The LITT process $[2,3]$ can be described via

$$
\begin{aligned}
\rho c_{p} \partial_{t} T-\nabla \cdot(k \nabla T) & =\xi_{b}\left(T_{b}-T\right)+\mu_{a}(\zeta) \varphi, \\
-\nabla \cdot(D(\zeta) \nabla \varphi) & =-\mu_{a}(\zeta) \varphi, \\
\partial_{t} \zeta & =-A \exp \left(-\frac{E_{a}}{R T}\right) \zeta
\end{aligned}
$$

with $T(0)=T_{0}, \zeta(0)=\zeta_{0}$ and the boundary conditions

$$
k \nabla T \cdot \mathbf{n}=\alpha_{\Gamma}\left(T_{\Gamma}-T\right), \quad D(\zeta) \nabla \varphi \cdot \mathbf{n}= \begin{cases}\frac{1-\beta}{\left|\Gamma_{\mathrm{rad}}\right|} q_{\mathrm{appl}} & \text { on } \Gamma_{\mathrm{rad}}, \\ 0 & \text { on } \Gamma_{\mathrm{cool}}, \\ -\frac{1}{2} \varphi & \text { on } \Gamma_{\mathrm{amb}}\end{cases}
$$

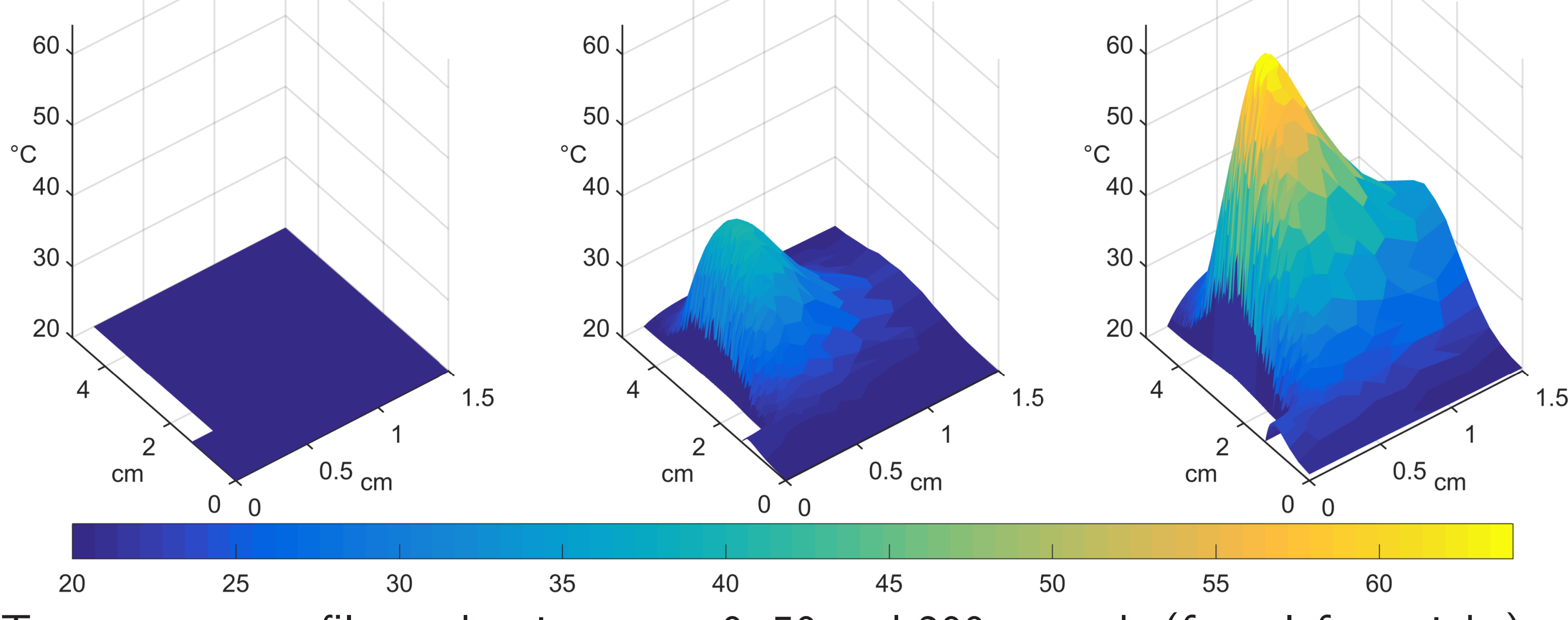

Temperature profile at the times $t=0,50$ and 200 seconds (from left to right).

\section{Space-Mapping}

The space-mapping technique $[1,4]$ aligns a (fast) coarse model $c: U_{c} \rightarrow \mathbb{R}^{m}$ with an (exact) fine model $f: U_{f} \rightarrow \mathbb{R}^{m}$ with the help of the space mapping function

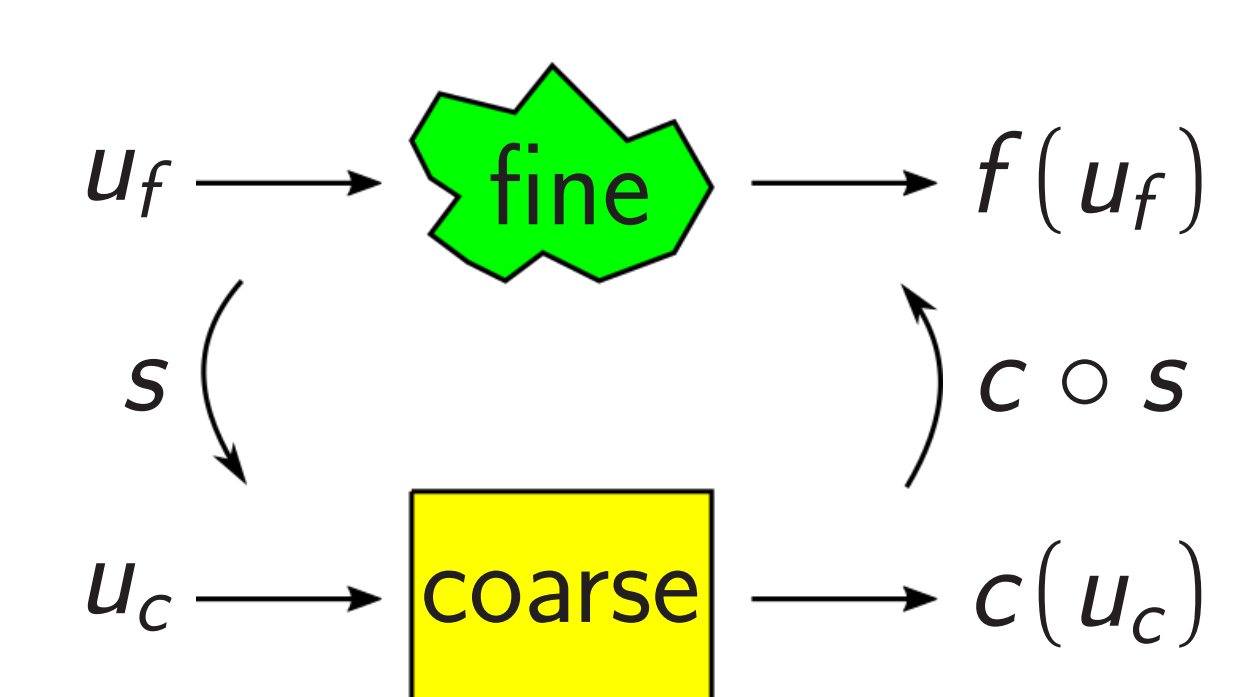

$$
\begin{aligned}
s: U_{f} & \rightarrow U_{c}, \\
u_{f} & \mapsto \underset{u_{c} \in U_{c}}{\operatorname{argmin}} \frac{1}{2}\left\|c\left(u_{c}\right)-f\left(u_{f}\right)\right\|^{2} .
\end{aligned}
$$

The aggressive space-mapping method solves the problem

$$
F\left(u_{f}^{*}\right)=s\left(u_{f}^{*}\right)-u_{c}^{*} \stackrel{!}{=} 0,
$$

where $u_{c}^{*}$ is the optimal solution of the coarse optimization problem

$$
u_{c}^{*}=\underset{u_{c} \in U_{c}}{\operatorname{argmin}} \frac{1}{2}\left\|c\left(u_{c}\right)-y_{d}\right\|^{2}
$$

with the desired state $y_{d}$.

\section{Coarse Model}

By reducing the coagulation effects to the scalar parameters $\bar{\mu}_{a}$ and $\bar{D}$, the nonlinear terms in (LITT) fall away, delivering the following linear approximation

$$
\begin{aligned}
\rho c_{p} \partial_{t} T-\nabla \cdot(k \nabla T) & =\xi_{b}\left(T_{b}-T\right)+\bar{\mu}_{a} \varphi, \\
-\nabla \cdot(\bar{D} \nabla \varphi) & =-\bar{\mu}_{a} \varphi .
\end{aligned}
$$

The parabolic characteristic of (sLITT) allows for a significant reduction in model complexity via model order reduction techniques, but the quality of the approximation itself deteriorates as the tissue properties noticeably change.

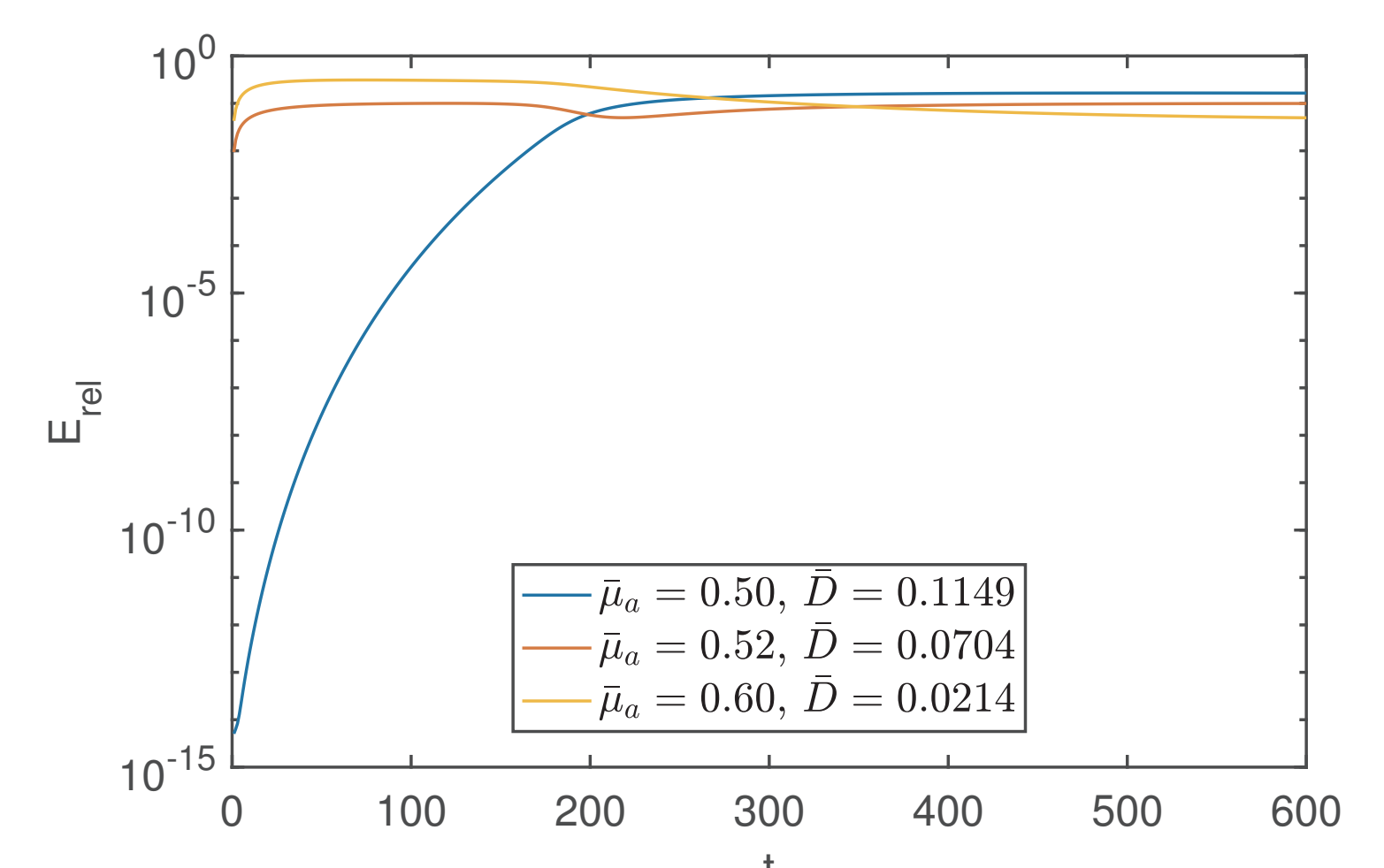

"Linearisation" is viable for short intervals.

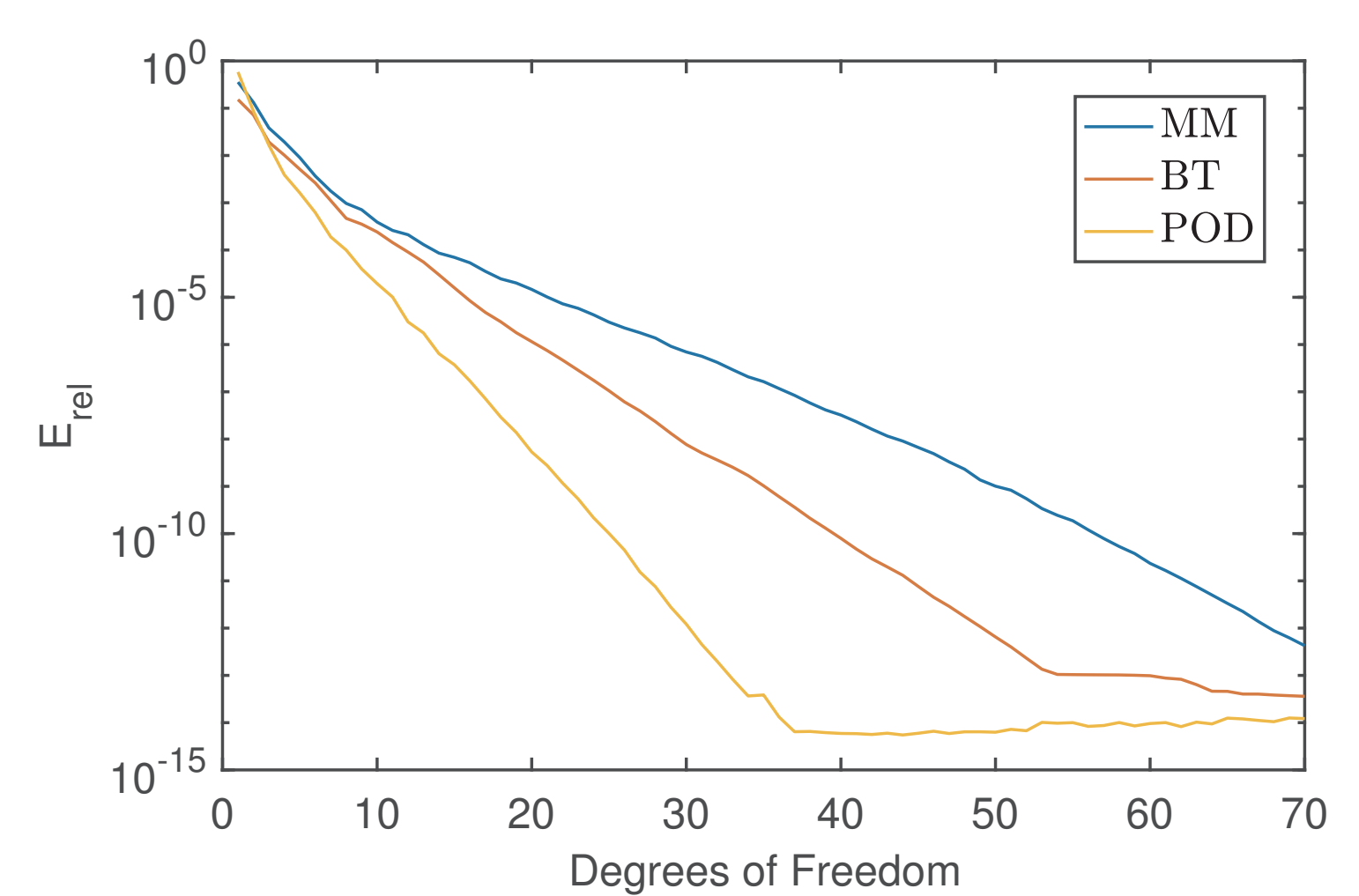

Full-order model consists of 1037 variables.

\section{Results}

The following optimal control problem attempts to reconstruct the laser power application that results in a given temperature progression:

$$
\min _{q_{\text {appl }}} J\left(T, q_{\text {appl }}\right)=\frac{1}{2}\left\|T-T_{d}\right\|_{L^{2}(Q)}^{2}+\frac{1}{2}\left\|T\left(t_{\text {end }}\right)-T_{d}\left(t_{\text {end }}\right)\right\|_{L^{2}(\Omega)}^{2}
$$
subject to (sLITT) and $0 \leqslant q_{\text {appl }}(t) \leqslant 30$ for all $t \in\left[0, t_{\text {end }}\right]$.

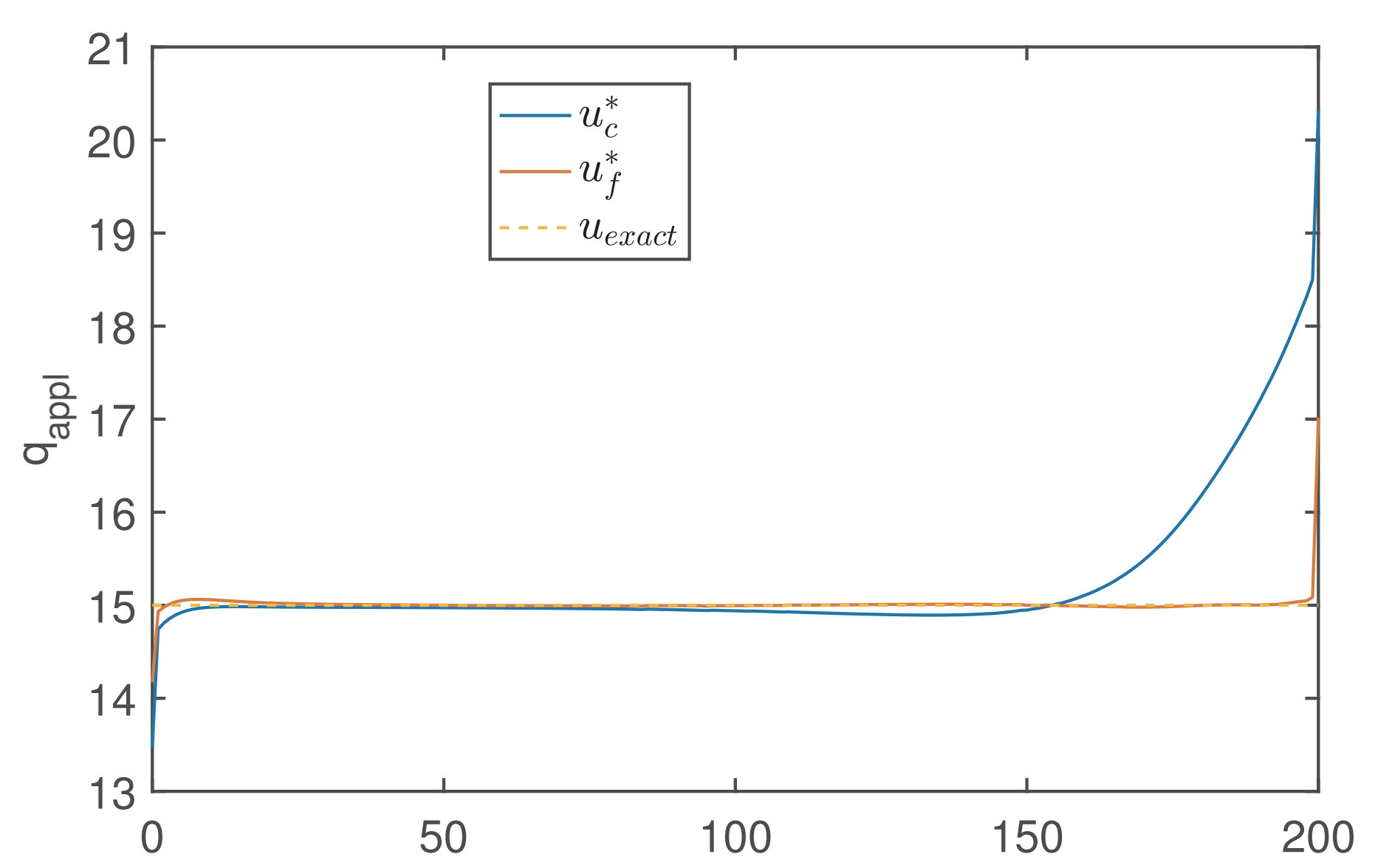

The space-mapping method manages to align the coarse model with the dynamics of the nonlinear model, avoiding direct optimization using the fine model.

With minimal effort, the aggressive space-mapping algorithm is able to attain a much better reconstruction of the temperature progression than the coarse optimization in just 10 iterations.

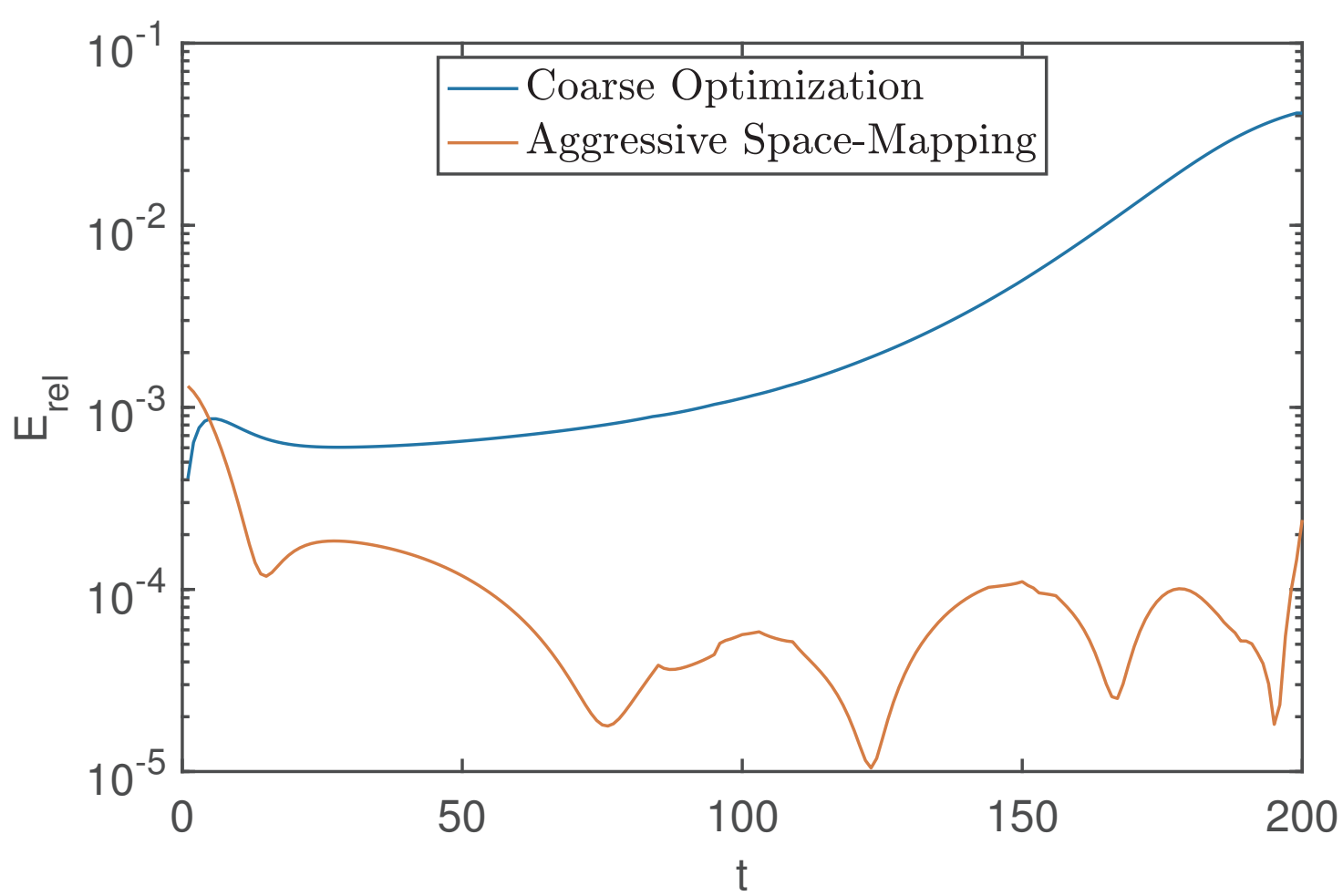

\section{Outlook}

- Improve the linear model by letting the parameters $\bar{\mu}_{a}$ and $\bar{D}$ vary with time (or depend on the temperature).

- Use a parametric model order reduction scheme in order to account for parameters $\bar{\mu}_{a}$ and $\bar{D}$.

- Fit the coarse model to the nonlinear model via a parameter identification problem.

- Expand the optimal control problem to take other attributes into consideration, e.g. amount of thermal energy introduced into the system or when coagulation occurs.

\section{References}

[1] M. H. Bakr, J. W. Bandler, K. Madsen, and J. Søndergaard. An introduction to the space mapping technique. 2001

[2] A. Fasano, D. Hömberg, and D. Naumov. On a mathematical model for laser-induced thermotherapy. 2010.

[3] F. Hübner, C. Leithäuser, B. Bazrafshan, N. Siedow, and T. J. Vogl. Validation of a mathematical model for laser-induced thermotherapy in liver tissue. 2017.

[4] N. Marheineke and R. Pinnau. Model hierarchies in space-mapping optimization: Feasibility study for transport processes. 2012. 